\title{
A de-mixing approach for the management of large negative peaks in wind tunnel data
}

\author{
François Rigo $^{\mathrm{a}, \mathrm{b}, \mathrm{c}}$, Thomas Andrianne ${ }^{\mathrm{a}}$, Vincent Denoël ${ }^{\mathrm{c}, *}$ \\ ${ }^{a}$ Wind Tunnel Lab, University of Liège, Belgium \\ ${ }^{\mathrm{b}}$ F.R.S.-FNRS, National Fund for Scientific Research, Belgium \\ ${ }^{\mathrm{c}}$ Structural \& Stochastic Dynamics, University of Liège, Belgium
}

\section{A R T I C L E I N F O}

\section{Keywords:}

Wind pressure

Peak factor

Non-gaussianity

Mixture distribution

Wind tunnel tests

Low-rise building

\begin{abstract}
A B S T R A C T
Large negative peak pressures commonly take place near the edges of buildings due to the presence of local corner vortices and impingement of shear layers. As a result, Probability Density Functions (PDF) of the measured pressure signals exhibit one or more components which contributes to the non-Gaussianity of the pressure loading. These mixed flows can be modeled with mixture models. Whenever several processes coexist, and when one of them is leading in the tail of the statistical distribution, it is natural to construct the extreme value model with only this process leading in the tail and not with the mixed observed pressures. In this paper, we propose a method that is based on the autocorrelation of the pressure coefficient to de-mix the measured signals. This information improves the de-mixing process where classical methods would struggle. Indeed, the two phenomena to be separated and identified might be characterized by significantly different time-scales, which are not reflected in the PDF. In this paper, the large negative pressures measured on a flat roof are analyzed and decomposed into two elementary processes, namely, the flapping corner vortex and the turbulent flow detaching from the sharp upstream edges. This paper finally shows that an accurate decomposition of the recorded pressures into their underlying modes provides a more meaningful evaluation of extreme pressures.
\end{abstract}

\section{Introduction and motivation}

Wind pressures on buildings are characterised by non-Gaussian stochastic processes. This fact has been supported for several decades by wind tunnel and on-site measurements (Newberry et al., 1974; Holmes, 1981; Peterka, 1983; Letchford and Mehta, 1993; Gioffrè et al., 2001; Sadek and Simiu, 2002). With the increasing sampling frequencies and denser pressure tap grids available today, larger extreme values can be captured in wind tunnel studies, which keeps offering an important interest to this problem (Amerio, 2017; Amerio et al., 2018; Huang et al., 2016; Rizzo et al., 2018). In many cases, the non-Gaussianity of the pressure field is attributable to large negative (suction) peaks in the separated-flow regions and on leeward walls of high-rise buildings. This non-Gaussianity makes the processing of wind tunnel data rather challenging in several aspects.

First, the statistical treatment of the peak pressure does not follow the usual procedure that is used for Gaussian processes (Davenport, 1964a), which would, otherwise results in significant discrepancies (Quan et al., 2009). This explains the development of numerous models aiming at capturing the non-Gaussian nature of the pressure field (Chen and Huang, 2009; Ding and Chen, 2014; Huang et al., 2017; Kwon and Kareem, 2009, 2011; Liu et al., 2017; Peng et al., 2014). Similarly, standard methods to estimate equivalent static wind loads such as the load-response correlation have difficulties to accurately reconstruct the envelope of structural responses (Blaise et al., 2017). Some equivalent static wind loads have, however, been designed to deal with the (possibly dynamic) response of structures to significantly non-Gaussian pressure fields (Blaise et al., 2016).

Second, it turns out that the events that are responsible for the very large negative peaks occupy a limited area in space (Amerio, 2017), usually smaller than the typical distance between two pressure taps, which stirs up interest in understanding the spatial coherence associated with these events. These two items and their consequences are briefly discussed next. Although the methodology presented in this paper is illustrated for very large negative and localized peaks, it could also be applied to mildly non-Gaussian processes with coherent structures being correlated along a wider area. It would offer a complementary outlook on the de-mixing possibilities offered by the independent component

\footnotetext{
* Corresponding author.

E-mail address: v.denoel@uliege.be (V. Denoël).
} 
analysis, see e.g. (Carassale and Brunenghi, 2012), and other source separation methods (Comon and Jutten, 2010). In any case, it is important to notice that a large amount of literature is available in wind engineering about the second-order decomposition of a wind pressure field. By second order, we mean the methods based on the covariance (zero-lag autocorrelation matrix), power spectral densities and cross-power spectral densities (Jolliffe, 2005). These methods are based on eigenvalue decomposition (Best and Holmes, 1983; Holmes et al., 1997), principal component analysis for the decomposition (Bienkiewicz et al., 1995; Baker, 2000; Solari et al., 2007), double decomposition (Tubino and Solari, 2005) and reconstruction (Kho et al., 2002) of the wind pressure field. All these methods are not suitable to decompose a significantly non-Gaussian pressure field into simpler components. Indeed, in statistics, the lower the considered moment, the closer to the bulk of the density. Hence, as soon as peak values and tail events are considered, higher statistics are required.

Pressures measured in a wind tunnel are used in the structural design with two major aims: (i) the determination of equivalent loads that can be used for the design of the main (Kareem and Zhou, 2003) and more locally important (Blaise and Denoël, 2013; Blaise et al., 2016) elements of the structural system and (ii) the design of cladding and other façade elements with respect to the local extreme pressures. The former question is not significantly affected by the non-Gaussian nature of the wind pressures. Indeed, evoking the central limit theorem, their imperfect correlation in space along the whole structure results in rather Gaussian base shear and overturning moment. Similarly, for the main elements of the bearing system, as soon as the influence line/surface affecting these elements is large enough, they tend to exhibit rather Gaussian responses. However, the occurrence of large localized negative peaks is decisive in the design of façade elements, whose sizes could be similar to the extent in space of these large suction peaks, which does not make them benefit from the central limit theorem.

Very large negative peak pressures have been recently reported, which triggers a certain concern in how to deal with these large negative pressures for the design of façade elements. These are traditionally designed in a static manner, which therefore means that they would have to withstand (in a static way) these large negative pressures. So far, values of pressure coefficients as large (in absolute value) as $c_{p}=-18$ (Lin et al., 1995), $c_{p}=-14$ (Amerio, 2017) or $c_{p}=-8$ (Blaise et al., 2017) have already been observed. Although they are hardly believable to any practitioner (bearing in mind the economic consequences of using such large pressure coefficients in a static design), the experimental value of these large numbers is demonstrated. They are substantially larger than typical pressure coefficients on buildings, and their treatment in the design process of cladding elements requires a specific attention. Because it is naturally difficult to design the cladding element on the basis of a very large peak pressure (along the entire element), several solutions have been imagined to define design forces in reasonable ranges. To consider the shorter space scale of these events, and the imperfect correlation in space (Denoël and Maquoi, 2012), or so-called size effect (Carassale, 2012; Blaise et al., 2014), is a first natural way to reduce the total resultant of wind pressures on a cladding element. In fact, this first option to deal with these large negative peaks dates back to the late 1970s. Because the allowable density of pressure taps was not fine enough to capture the spatial distribution of the extreme suction events, the pioneers in wind engineering have proposed to connect the time and space scales of the wind pressure, through a relation of the type $T=$ $4.5 L / V$, where $L$ is a characteristic length; see works of Newberry (Newberry et al., 1974), then Lawson (Lawson and. Lawson, 1980), Cook (1986) and Holmes (1997). In these works, the assumption of frozen turbulence (Dyrbye and Hansen, 1996) is already predominant. It connects the time and space scales, which seems acceptable as long as the wind field of the atmospheric boundary layer is concerned and is stationary (in time) and homogenous (in space). The first attempts at modeling the time-space dependency in this way seem to be attributable to Davenport (1964b), and indeed related to a turbulent wind field. Then, when analyzing the pressures on the Royex building, Newberry (Newberry et al., 1974) founds a similar relation for the pressure field, in areas of the building which are located close to the center of the building faces, but not close to edges. These two observations have inspired the following generation of researchers in using a frozen turbulence approach to model the stochastic wind fields, which has been adopted by modern design codes (Cook, 1986; Eurocode, 1991). Furthermore, most of the above-mentioned references have considered a real exponentially decreasing co-spectrum (Dyrbye and Hansen, 1996). In this work, we further justify why it is desired not to base our work on a time-space modeling of the wind pressure and explain why an analysis of the pointwise data (i.e. a set of stochastic processes rather than a stochastic field) still makes sense today. This paper is therefore limited to the analysis of the non-Gaussian nature of the wind pressure at a single measurement point.

Non-Gaussian stochastic processes are usually modeled with a nonGaussian probability density function (PDF) and a power spectral density (PSD) (Grigoriu, 1998). This is by the way the usual information that is considered today to generate samples of non-Gaussian processes (Gioffre and Grigoriu, 2005; Gurley and Kareem, 1999). The non-Gaussian nature of the process can be revealed by the PDF, as well as the first few statistical moments. In other models, the bispectrum of the load is also modeled (Shields et al., 2011), which plays a major role as soon as the dynamics of the structural system cannot be neglected (Denoël, 2011). When very large negative peaks are of concern, the wind pressure typically features two distinct and interacting phenomena (Cook, 2016; Gioffre and Grigoriu, 2016). In worse cases (of large non-Gaussianity), the pressure distribution can even become bimodal which immediately calls for a separation of the two different phenomena (Cook, 2016; Gioffre and Grigoriu, 2016). Existing procedures to decompose a time series into two components are typically based on the PDF of the measured pressure (Cook, 2016), while other blind source separation techniques (Comon and Jutten, 2010) would typically require several combinations of the independent processes in order to be able to decompose them. Observing that the timescales associated with the two mixed components are different (Rigo et al., 2018) (typically slow background turbulence and fast shedding/flapping), in this paper, we propose to perform the de-mixing operation with the help of the autocorrelation of the measured pressure. This decomposition of the wind pressure at a given location provides a clear picture of the two or more competing sources of activity leading to the (mixed) measured pressure.

In passing, it is observed that the cubic translation model (Winterstein, 1987) that is widely used to model extremes of wind pressure data, requires to meet some monotone conditions (Peng et al., 2014). The limit corresponds to the occurrence of an inflection point in the cubic transformation. This situation naturally tends to occur when the PDF of the wind pressure is bimodal. This makes the translation model inapplicable in these cases. To compensate for this limitation, several modifications have already been proposed, see e.g. (Peng et al., 2014); although being driven by a search of pragmatic procedures, they are usually not based on physics. Alternatively, we suggest dealing with these processes failing to satisfy the monotone condition, by first de-mixing the wind pressure process, and then applying a cubic translation model to each component. An example given in this paper shows that this approach significantly extends the applicability and accuracy of the cubic translation model.

The paper is organized as follows. First, a short discussion on the stationarity in time and space of the wind pressure field is given. Then, the novel algorithm to de-mix pointwise pressure data, on the basis of the PDF and the different timescales in the process, is presented. The procedure is illustrated with a set of pressure data measured on a flat roof at $45^{\circ}$ incidence, which is known to produce corner vortices. An illustration shows that the proposed algorithm is able to efficiently de-mix the two components. Last but not least, two examples of added value of the proposed de-mixing are shortly presented. The first one concerns the applicability of the cubic translation model; the second concerns the interpretation of the total pressure on a cladding as a sum of a slow 
(quasi-static) turbulence and a fast (impulsive) sequence of suction peaks.

\section{Homogeneity in space and stationarity in time}

A large literature about the distribution in space of the wind velocity or pressure is based on the concept of admittance (Holmes, 1997; Denoël and Maquoi, 2012), frozen turbulence (Dyrbye and Hansen, 1996) or similar models relating time and space with a characteristic velocity. With a little perspective, it appears obvious that this equivalence between time and space scales ought not be used in the areas of the structure where the wind pressure is not stationary in time or non-homogenous in space. The point followed in this paper is that this is precisely in these areas that the pressures are significantly non-Gaussian. Indeed, although the wind pressure field could be considered as statistically stationary in time even in the areas of large non-Gaussianity (to be detailed next), it is certainly not homogenous in space, in general, and especially where significantly non-Gaussian pressure fields are observed, e.g. in the neighborhood of the edges of a building. In these areas, the pressure field is non homogenous in space, i.e. the statistics of the wind pressure change from place to place. For instance, the first rank statistics (mean, standard deviation, skewness, etc.) exhibit large gradients, which is enough to classify the wind pressure field as non-homogenous in space. On the opposite, the wind pressures are stationary in time, i.e. the statistics are independent of the origin of time; in other words, the first rank statistics are independent of time. This is even better, wind pressures are typically ergodic processes in time, which means that the statistics performed through the probability space are equally estimated by time averages (Papoulis, 1991) (p. 427), or, said differently, a very long time series could be used to estimate the statistics of the stochastic process. This simple argument explains why any attempt at modeling the non-homogenous nature of wind pressures with a universal equivalent time-space representation, as in the TVL approach, or with an admittance expressed as a function of $\omega \ell / U$ (with $\omega$ the inverse of a timescale, $\ell$ a length scale and $U$ the mean wind speed), would naturally fail. This fact has been experimentally shown by Amerio et al. (Amerio, 2017; Amerio et al., 2018), by observing that it is impossible to determine a unique coefficient in the TVL equation, that would be universal in predicting the resultant pressure over a certain area located close to an edge of a building, and in various conditions of wind exposure.

In this paper, the variability in space of the wind pressure field is not discussed and we propose to focus on the pointwise time domain data. We first recognize that in many wind engineering problems, the nonGaussianity of the pressure is associated with bimodal or multimodal distributions. Being aware of this and having observed that the two underlying components of the pressure are associated with two different timescales, an autocorrelation-based de-mixing method is suggested to improve the standard conditioning of de-mixing techniques.

\section{The considered problems}

The autocorrelation-based de-mixing algorithm described in Section 4 shall be efficient in decomposing the wind pressure at a given point into several components which evolve on different timescales, and which could, contrarily to existing de-mixing methods, be able to deal either with components having very similar PDFs, either with components whose mixing weights are significantly different. So the first considered problem is the de-mixing of such pressure time series.

This problem being solved, the statistics of the different components in the pointwise wind loading are known, mode by mode. This offers an interesting outlook on many connected problems.

A first adjoint problem concerns the estimation of the extreme value distribution of the pressure coefficient. Once the two (or more) modes are separated, it is straightforward to consider the mode that governs the tail distribution and only consider this mode to determine the distribution of the peak pressure. As an example, a cubic translation model would be simple to use on this mode only, while inappropriate for the mixed process (because the monotone condition is usually not met for mixed processes). The de-mixing offers there a broader range of application of the simple and convenient tools that have been massively used in wind engineering. This question is addressed by means of an example is Section 5 .

A second adjoint problem concerns the design of cladding elements. This question is briefly touched in Section 6. Having decomposed the pressure into two components, a fast shedding/flapping with large negative peaks and a slow background turbulent flow, it becomes natural to consider a dynamic analysis of the cladding element. In particular, it is known that the structural response to a fast impulsive load is governed by the momentum of the load and might be associated with a significant reduction of the pressure to be considered in an equivalent static analysis. This approach appears therefore as a natural alternative to the TVL method and the likes, in order to process the large negative pressure peaks into realistic static pressure coefficients.

\section{The autocorrelation-based de-mixing algorithm}

\subsection{The mixture process model}

This motivates the need to develop a procedure to decompose the measured wind pressure field into elementary processes. De-mixing random variables and stochastic processes is a mathematical problem that finds applications in many fields of sciences (Comon and Jutten, 2010). In wind engineering, separation techniques have already been used to analyze wind speed especially in the field of wind energy production (Kollu and Pakkurthi, 2012; Yu et al., 2013), but also for the determination of design wind speeds (Cook, 2016). For instance, Cook (2016) proposes the Skew Gaussian Exponential Mixture Model to model the wind pressure data as a mixture of variables following one or several skew-Gaussian distribution(s) and one or several skew hyperbolic secant distribution(s). This model is exploited here with the major difference that we specifically consider random processes instead of variables, stressing out that the mixing process is a random process itself and not a random variable. In the considered model, the value of the pressure coefficient is expressed as

$c_{p}(t)=w_{1}(t) c_{p, 1}(t)+w_{2}(t) c_{p, 2}(t)$

where we have limited the model to two components, which is justified by the fact that we would like to model two physical processes in the wind flow; following Cook's approach, they are modeled as two skewGaussian components. The mixing processes $w_{1}(t)$ and $w_{2}(t)$, such that $w_{1}(t)+w_{2}(t)=1$, are weighting factors between the two underlying components $c_{p, 1}(t)$ and $c_{p, 2}(t)$. The stochastic process $c_{p}(t)$ is called mixture process model since $w_{1}(t)$ and $w_{2}(t)$ are equal to either 0 or 1 at each time. This choice of model can therefore be seen as a very specific case of a weighted combination (where $w_{1}(t)$ and $w_{2}(t)$ would belong to $[0,1])$. In a mixture process, the pressure coefficient $c_{p}$ is drawn from one of the two distributions of $c_{p, 1}$ or $c_{p, 2}$ at each time. Whether a realization is drawn from one or the other distribution is also correlated in time, since $w_{1}(t)$ and $w_{2}(t)$ have some finite memory. This makes this model substantially different from existing mixture models for variables which have no memory. In fact, the memory of the mixing processes is rather long since many consecutive samples need to be taken out of a distribution, either $c_{p, 1}$ either $c_{p, 2}$, in order to not alter the continuity of the process (Fig. 1). It makes it also different from the more general weighted combination of the two underlying processes $c_{p, 1}(t)$ and $c_{p, 2}(t)$, for which $w_{1}(t)$ and $w_{2}(t)$ belong to the full interval $[0,1]$. Again, following Cook's approach and the new recent trends in wind engineering, the mixture model is chosen because it offers simple closed form expressions, as seen next, whereas the general weighted combination model would turn into more accurate but cumbersome equations.

Assuming that the mixing processes $w_{1}(t)$ and $w_{2}(t)$ and the two 


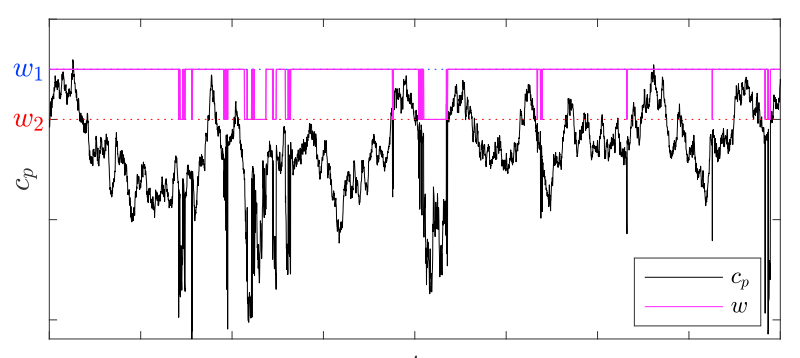

$t$



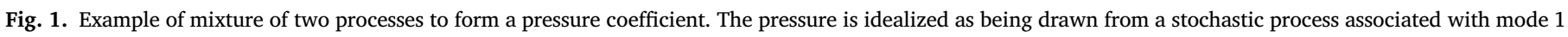
for a certain duration, that a little later, as being drawn from a stochastic process associated with mode 2 .

underlying processes $c_{p, 1}(t)$ and $c_{p, 2}(t)$ are statistically independent, it is possible to prove (see Appendix A) that the PDF of the pressure coefficient is given by

$p_{c_{p}}(x)=\bar{w}_{1} p_{c_{p, 1}}\left(x ; \boldsymbol{\pi}_{1}\right)+\bar{w}_{2} p_{c_{p, 2}}\left(x ; \boldsymbol{\pi}_{2}\right)$

where $\bar{w}_{1}$ and $\bar{w}_{2}$ are the average values of $w_{1}(t)$ and $w_{2}(t)$, i.e. the average mixing ratios and where $p_{c_{p, i}}\left(x ; \pi_{i}\right)$ with $i \in\{1,2\}$ are the (stationary) skew-Gaussian PDF of the underlying processes $c_{p, 1}(t)$ and $c_{p, 2}(t)$. They are given by

$p_{c_{p, i}}\left(x ; \boldsymbol{\pi}_{i}\right)=\frac{2}{s_{i}} \varphi\left(\frac{x-m_{i}}{s_{i}}\right) \Phi\left(\alpha_{i} \frac{x-m_{i}}{s_{i}}\right)$

where $\varphi$ and $\Phi$ are the standard Gaussian PDF and cumulative density function (CDF), $m_{i}$ a position parameter, $s_{i}$ a scale parameter and $\alpha_{i}$ a skewness parameter (Azzalini and Valle, 1996). To simplify notations, these three parameters are gathered in $\pi_{i}=\left\{m_{i}, s_{i}, \alpha_{i}\right\}$ for each of the two underlying processes. They are related to the mean $\mu_{i}$ and variances $\sigma_{i}^{2}$ of $c_{p, i}(t)$, for $i \in 1,2$, by

$\mu_{i}=m_{i}+\frac{s_{i} \alpha_{i} \sqrt{2}}{\sqrt{\pi}\left(1+\alpha_{i}\right)^{1 / 4}} \quad ; \quad \sigma_{i}^{2}=s_{i}^{2}\left(1-\frac{2 \alpha_{i}^{2}}{\pi\left(1+\alpha_{i}^{2}\right)}\right)$

The average of the mixed pressure coefficient is given by $\mu_{x}=\bar{w}_{1} \mu_{1}+$ $\bar{w}_{2} \mu_{2}$, while its variance is given by $\sigma_{c_{p}}^{2}=\bar{w}_{1} \sigma_{1}^{2}+\bar{w}_{2} \sigma_{2}^{2}+\bar{w}_{1} \bar{w}_{2}\left(\mu_{1}-\mu_{2}\right)^{2}$. Notice that when $\left|\mu_{1}-\mu_{2}\right| \ll\left\{\sigma_{1}^{2}, \sigma_{2}^{2}\right\}$, the variance of the mixture process is just a weighted combination of the variances in the two components, exactly as it is the case for the average value.

Furthermore, under the assumptions that (i) the characteristic timescale of the mixture process is longer than the characteristic timescales in the two underlying processes $c_{p, 1}(t)$ and $c_{p, 2}(t)$ and (ii) $\left|\mu_{1}-\mu_{2}\right| \ll\left\{\sigma_{1}^{2}\right.$, $\left.\sigma_{2}^{2}\right\}$, the autocorrelation function of $c_{p}(t)$ may be approximated by (see Appendix A)

$R_{c_{p}}(\Delta t)=\bar{w}_{1} R_{c_{p, 1}}(\Delta t)+\bar{w}_{2} R_{c_{p, 2}}(\Delta t)$

where $R_{c_{p, 1}}(\Delta t)$ and $R_{c_{p, 2}}(\Delta t)$ represent the autocorrelation functions of $c_{p, 1}(t)$ and $c_{p, 2}(t)$.

\subsection{Classical de-mixing approach}

A classical de-mixing approach, such as that presented in (Cook, 2016) consists in adjusting the model parameters $\bar{w}_{1}, \pi_{1}$ and $\pi_{2}$ in order to minimize the residuals between the PDF of the mixture model (2) and a non-parametric estimate $\tilde{p}_{c_{p}}$ of the PDF of the experimental data. Notice that $\bar{w}_{2}$ is omitted in the procedure since $\bar{w}_{2}=1-\bar{w}_{1}$. Formally, collecting the 7 parameters of the model in $\pi=\left\{\bar{w}_{1}, \pi_{1}, \pi_{2}\right\}$, the statistical properties of the two underlying components are obtained by solving the following unconstrained minimization problem $\widehat{\boldsymbol{\pi}}=\underset{\pi}{\operatorname{argmin}}\left(p_{c_{p}}(x ; \boldsymbol{\pi})-\tilde{p}_{c_{p}}\right)^{2}$

Many optimization packages are available in shared libraries; the implementation of such a minimization procedure is therefore not discussed. For the selected method to work efficiently, it is necessary that the minimization problem be well-posed. Specifically, the well-posedness of the problem depends on the gradients of the objective function $F(\boldsymbol{\pi})=$ $\left(p_{c_{p}}(x ; \pi)-\tilde{p}_{c_{p}}\right)^{2}$ with respect to the unknown parameters $\pi$. Intuitively, the problem is well conditioned when the two components are well separated and create a bimodal distribution. On the contrary, for mixed components having close average (position) parameters, and similar standard deviations, the de-mixing is more difficult. In the limit case of a mixture of two identical underlying distributions, it is observed that $\partial_{\bar{w}_{1}} F(\pi)=0$ since, in this case, $p_{c_{p, 1}}=p_{c_{p, 2}}$ and $\bar{w}_{1}=1-\overline{\boldsymbol{w}}_{2}$. This means that $\bar{w}_{1}$ has no influence on the objective function and cannot be determined. It is, however, important to separate the two existing processes since they might exhibit different dynamics and hence different extreme value statistics. Anyways, in all other cases of close average values, compared to the spread of the distributions of the underlying processes, classical methods based on the PDF (first-rank information only) tend to lack efficiency.

\subsection{Proposed de-mixing approach}

The difficulty to separate two mixed random processes with similar statistical properties might be avoided if they have significantly different dynamics/timescales. And this is the case in many wind engineering problems! Close to edges of buildings, two phenomena are involved in the pressure, a mixture between a slow background turbulence $c_{p, 1}$ and a fast shedding $c_{p, 2}$, which is visible for instance in the autocorrelation function of the mixed process, which is the only quantity that is measured, see Fig. 2 (bottom). In a similar fashion, corner vortices, horseshoe vortices and Von Karman vortices (Gharib et al., 1998; Hunt et al., 2000) detaching around bluff bodies in a turbulent flow are usually associated with shorter timescales, i.e. fast dynamics.

Fig. 2 shows for instance a typical autocorrelation function of the pressure coefficient measured on the root of a building (details of the wind tunnel setup are coming in section 4.4). The breakpoint in the autocorrelation function reveals the transition from the fast shedding of the corner vortices and the slow turbulent component. With this in mind, it is suggested to split the autocorrelation of the pressure coefficient into two elementary contributions. Assuming that the mixing process is slow and that the two elementary processes have close average values (precisely where classical models are at stake), the autocorrelation is simply given by Equation (5). With this in mind, it is proposed to approximate the autocorrelation of the measured pressure coefficients $\tilde{R}_{c_{p}}(\Delta t)$ with a weighted combination of two exponentially decreasing functions,

$R_{c_{p}}(\Delta t)=\bar{w}_{1} \sigma_{1}^{2} e^{-\Delta t / T_{x, 1}^{\star}}+\bar{w}_{2} \sigma_{2}^{2} e^{-\Delta t / T_{x, 2}^{\star}}$ 

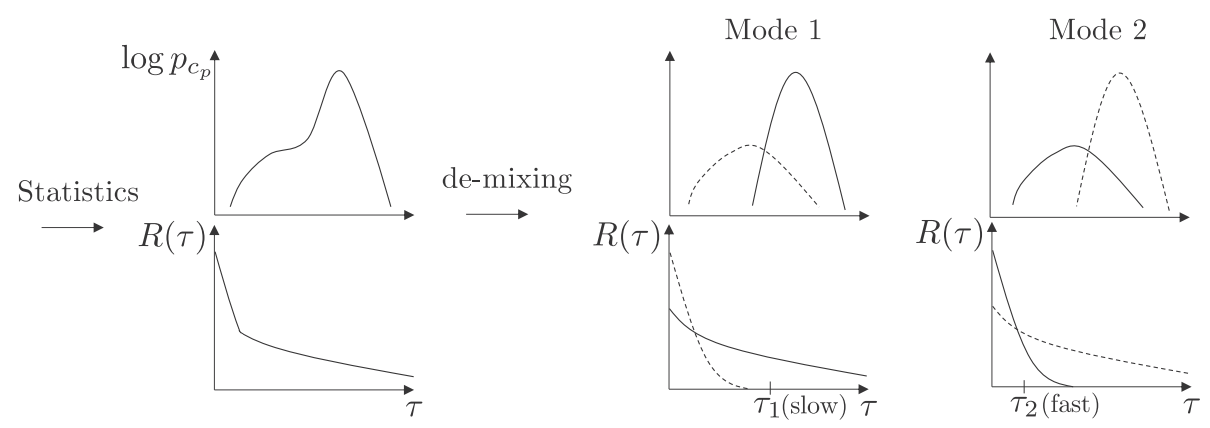

Fig. 2. Autocorrelation-aided de-mixing methodology.

where $T_{x, 1}^{\star}$ and $T_{x, 2}^{\star}$ are the characteristic timescales of the two underlying components. The adjustment of the mixture process model (including the timescales information coming from the autocorrelation) is then performed in two steps. First, solve

$\widehat{\boldsymbol{\psi}}=\underset{\psi}{\operatorname{argmin}}\left(\bar{w}_{1} \sigma_{1}^{2} e^{-\Delta t / T_{x, 1}^{\star}}+\bar{w}_{2} \sigma_{2}^{2} e^{-\Delta t / T_{x, 2}^{\star}}-\tilde{R}_{c_{p}}(\Delta t)\right)^{2}$

where $\boldsymbol{\psi}=\left\{\bar{w}_{1} \sigma_{1}^{2}, \bar{w}_{2} \sigma_{2}^{2}, T_{x, 1}^{\star}, T_{x, 2}^{\star}\right\}$ collects the four parameters of the second-rank problem. The two timescales $T_{x, 1}^{\star}$ and $T_{x, 2}^{\star}$ are important to understand the physics of the flow around the considered body but are not used in the following statistical treatment. On the contrary, the two weighted variances $\bar{w}_{1} \sigma_{1}^{2}$ and $\bar{w}_{2} \sigma_{2}^{2}$ are used to constrain the first-rank optimization problem, which now reads

$\widehat{\boldsymbol{\pi}}=\underset{\pi ; \bar{w}_{i} \sigma_{i}^{2} \text { known }}{\operatorname{argmin}}\left(p_{c_{p}}(x ; \boldsymbol{\pi})-\tilde{p}_{c_{p}}\right)^{2}$.

One way to transform this constrained into unconstrained optimization problem is to write the objective function $F(\boldsymbol{\pi})$ as a function of 5 parameters only, for instance by expressing $\sigma_{i}, i \in\{1,2\}$, and hence $s_{i}^{2}$ via (4), as a function of the known values of $\bar{w}_{i} \sigma_{i}^{2}, \widehat{\psi}_{1}$ and $\widehat{\psi}_{2}$, while keeping $\bar{w}_{1}$ in the list of parameters to adjust. In this approach, the final list of unknown parameters is limited to $m_{1}, m_{2}, \alpha_{1}, \alpha_{2}$ and $\bar{w}_{1}$.

Fig. 2 summarizes the autocorrelation-aided de-mixing methodology into two modes. The PDF and autocorrelation function of the time series can be computed and used to split the statistics into two elementary components. Although the sketch illustrates a time series with two obvious components, the proposed method is also applicable to more pathological cases.

\subsection{Illustration of the de-mixing algorithm}

4.4.1. Wind tunnel setup and flow analysis

The setup made by Blaise et al. (2017) consists of a square plan-form low-rise building (sides of $45 \mathrm{~m}$ and a height of $25 \mathrm{~m}$ ), uniformly instrumented by 121 pressure taps in a quarter of its roof (see Fig. 3(b)), sampled at $f_{s}=500 \mathrm{~Hz}$. The model is placed at an incidence of $45^{\circ}$ in the atmospheric boundary layer test section of the wind tunnel (WT) of the University of Liège (Fig. 3(a)). This direction is fixed to obtain symmetry in the complex nature of the corner vortices developed at the edges (Kawai and Nishimura, 1996).

The atmospheric boundary layer test section creates a turbulent wind of category III according to the Eurocode (Liu et al., 2017), with $z_{0}=0.3$ $\mathrm{m}$ and $z_{\min }=0.5 \mathrm{~m}$. The mean velocity (Equation (10)) and turbulence intensity (Equation (11)) profiles are presented in Fig. 4(a-b).

$U_{\infty}(z)=0.19\left(\frac{z_{0}}{0.05}\right)^{0.07} \ln \left(\frac{z}{z_{0}}\right) U_{r e f}$

$I_{u}(z)=\frac{1}{\ln \left(\frac{z}{z_{0}}\right)}$

Pressure taps are linked to the scanner using pneumatic connection (vinyl tubes) of internal diameter of $1.32 \mathrm{~mm}$ and length $600 \mathrm{~mm}$. The static pressure is well measured but unsteady components have to be dynamically corrected, thanks to the theoretical formulation of Bergh and Tijdeman (1967) and the experimental apparatus of Rigo (2017). Fig. 4(c) presents the transfer function, in amplitude and phase, necessary to correct measurements, until the sampling frequency of $500 \mathrm{~Hz}$. This calibration has to be carefully performed as the tube has a direct effect on the pressure amplitude and thus the peak values.

The geometric scaling is $\lambda_{L}=1 / 100$. The Reynolds condition would require a velocity scaling of $\lambda_{U}=\lambda_{L}^{-1}=100$, which would impose WT speed of about $1000 \mathrm{~m} / \mathrm{s}$, impossible. The Reynolds dependency is more critical for smooth and mainly circular shapes such as cylinder or sphere. When the flow is completely separated and turbulent, the Strouhal scaling is preferred. Because of WT performances, the velocity scaling is chosen as $\lambda_{U}=1 / 3.5$. The Strouhal condition imposes $\lambda_{T}=\lambda_{L} / \lambda_{U}=1 /$ 28.6 (time). Every measurement is converted in full scale. A total of $13 \mathrm{~h}$ of measurement has been taken in the WT, corresponding to $371.8 \mathrm{~h}$ full
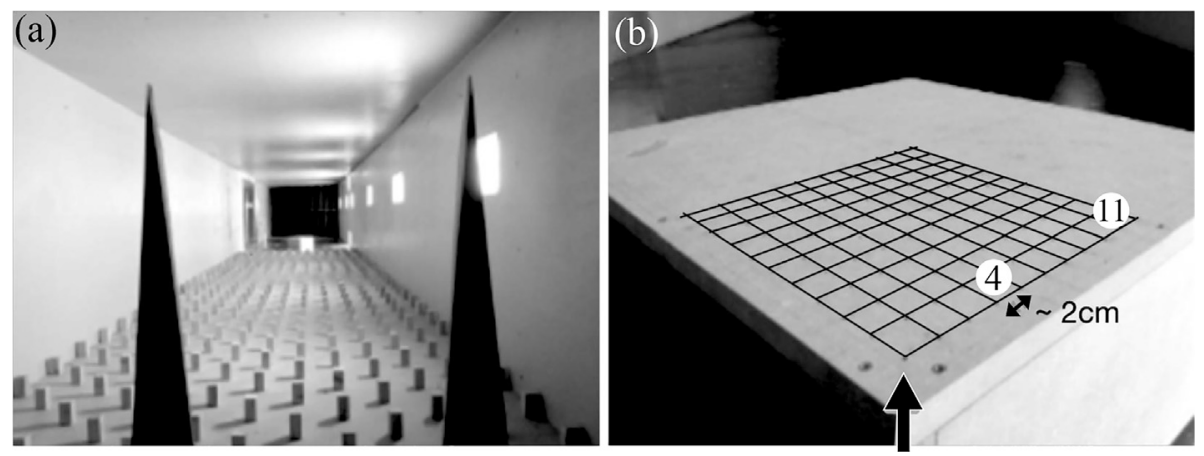

Fig. 3. (a) Model inside the WT, (b) location of pressure taps on the model (wind direction fixed at $45^{\circ}$ ). 



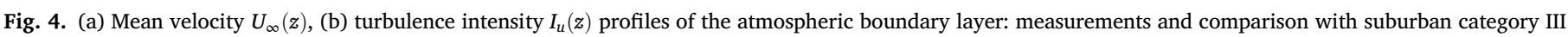

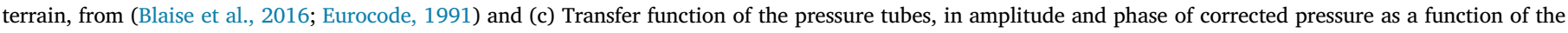
frequency (Bergh and Tijdeman, 1967; Rigo, 2017).

scale. This long information allows to compute precise PDF tail, necessary to study the extreme values. In the following, statistics are presented in a non-dimensional way: position $(\xi, \eta)=(x / L, y / L)$ and pressure coefficient $c_{p}=\frac{p-p_{\infty}}{\frac{1}{2} \rho U_{\infty}^{2}}$, with the windspeed $U_{\infty}$ taken at the height of the building ( $z=25 \mathrm{~m}$ in full scale). The Reynolds number of the WT model is $6.8 \times 10^{5}$.

The flow around this low-rise building is characterized by corner vortices, that roll in a cone shape. The pressure is mainly negative because of the separated nature of the flow $\left(\mu\left(c_{p}\right)\right.$ in Fig. 5(a)) and is the most negative where it starts to separate, just after the edges, on the corner. The two cones on both corners have a main axis, recognizable by the ridge lines of the standard deviation of the pressure coefficient $\sigma\left(c_{p}\right)$, in Fig. 5(b). The non-Gaussianity happens in the corner vortices boundaries, where $\gamma_{3}\left(c_{p}\right)$ and $\gamma_{e}\left(c_{p}\right)$ are the highest. For a Gaussian process, $\gamma_{3}=$ 0 and $\gamma_{4}=3$. The excess $\gamma_{e}=\gamma_{4}-3=0$. On Fig. 5, a lower right triangle appears systematically, with low statistics values. This region is mainly Gaussian, with a low dispersion and mean pressure value. Physically, the flow in this region is the case of a simple turbulent flow on a flat plate. This region is not studied here, since it has smaller extreme values and represents less interest compared to the complex flow in corner vortices.
As suggested by Kawai (Kawai and Nishimura, 1996), there is one main vortex at the corner, but a secondary small one develops just next to the edge, below the main vortex. Pressure taps in this setup were not close enough to the edge to capture this secondary vortex well. Nevertheless, in Fig. 5(c), $\gamma_{3}\left(c_{p}\right)$ increases just next to the edge, at $(\xi, \eta)=(0.05$ 0.4 ), suggesting the presence of another vortex. Banks (Banks et al., 2000) also suggested that the magnitude of the suction peak is inversely proportional to the size of the vortex and is the highest close to the edge. Moreover, increasing turbulence is moving the mean vortex core position closer to the leading edge. By looking at skewness and kurtosis values, each pressure distribution in the corner vortices has a negative skewness (negative extreme pressure events) and a positive excess kurtosis (called softening process, the PDF has a flatter shape compared to a Gaussian process). Fig. 5(e) shows pressure coefficient signals at taps 4 (near the roof edge), 28 (inside the corner vortex) and 50 (roof center). Tap 4 shows very negative pressure (up to $c_{p}=-8$ ) with an asymmetry in the signal (negative $\gamma_{3}$ ). Tap 28 shows lower negative peak pressure but with a higher occurrence, leading to a higher asymmetry and more negative $\gamma_{3}$. These two taps are highly non-Gaussian and interesting to analyze deeper, compared to tap 50 with a low Gaussian (symmetric) variation


(e)

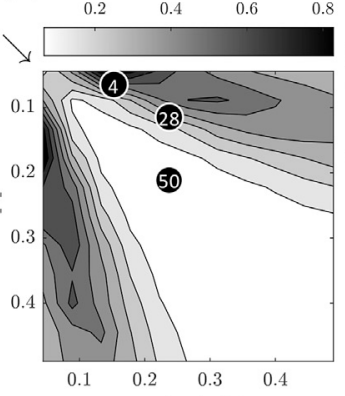

(d)
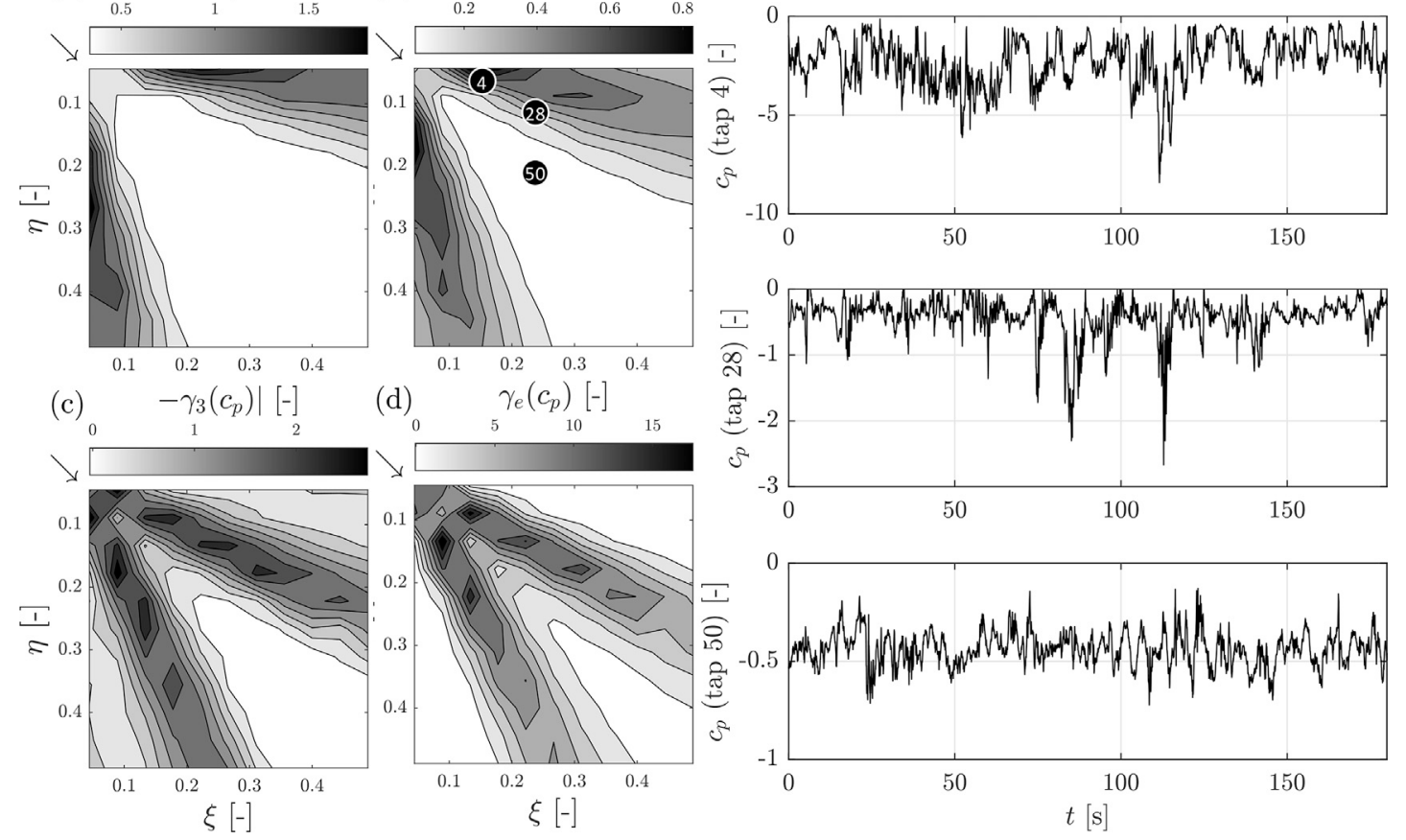

Fig. 5. Map of (a) mean, (b) standard deviation, (c) skewness, (d) kurtosis (excess) of all pressure taps and (e) example of time signals at taps 4,28 and 50. 

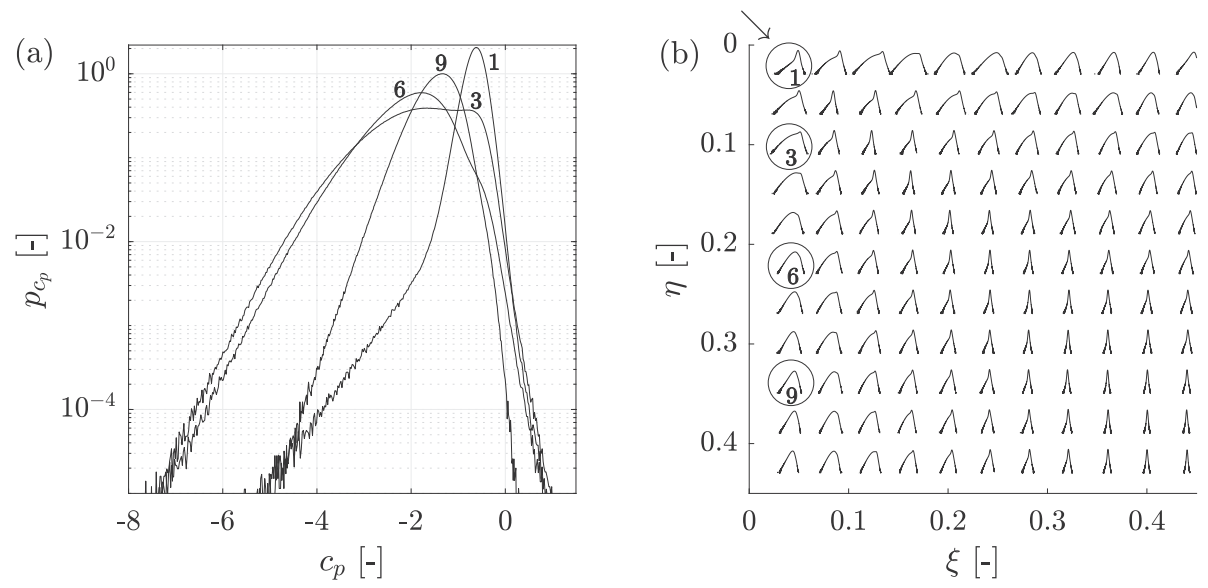

Fig. 6. Map of PDF of all taps on the roof (b) and focus on taps 1, 3, 6 and 9 (a).

around its mean $\mu\left(c_{p}\right)=-0.5$.

Fig. 6(b) gives a quick qualitative view of the PDF of pressure coefficients at all 121 pressure tap locations. Each PDF is represented in log scale. The same scaling is used for all of them in order have a comparative view of the dispersion and asymmetry of PDFs. Those in the zones affected by corner vortices are highly non-Gaussian and two modes (two bumps) are clearly identifiable. Fig. 6(a) shows the variation of the relative importance of the two bumps when moving along the edge of the roof (taps 1, 3, 6 and 9). This motivates the discussion of the next paragraph.

\subsubsection{Classical de-mixing}

Fig. 7(a) shows the result of the decomposition in modes of the PDF at pressure tap 4 . The two skew-Gaussian modes are easy to identify since two bumps in the PDF are clearly distinguishable. In this case, the classical de-mixing is accurate and does not require any consideration of the timescales in the two modes (or their autocorrelation) in order to de-mix them. Fig. 7(b) shows the same result but for pressure tap 8, for which the decomposition into two modes is less obvious. In that case, the classical de-mixing approach, based on the PDF only, is indeed able to find a possible decomposition; in fact the objective function is very flat in the neighborhood of the optimum, which indicates the large sensitivity of the method to small changes in the data.

\subsubsection{Proposed de-mixing}

Fig. 8(a) shows the PDF at tap 11. Modes are almost superimposed and very difficult to identify with the classical de-mixing approach (same as pressure tap 8 discussed above). On the opposite, the autocorrelation, shown in Fig. 8(b) has two obvious timescales. This indicates that, although the two elementary contributions we are seeking to de-mix have very similar distributions (1st rank properties), they are associated with different timescales (2nd rank properties). Following the proposed decomposition, the autocorrelation is split into two components. The intercepts of the two components are used to determine the weighted variances in each mode, which are, in turn, exploited to constrain the optimization problem and the pressure coefficient at tap 11 can be robustly decomposed into two modes.

\subsubsection{Required duration of the time series}

The results presented so far have been obtained from very long time series, covering in total $370 \mathrm{~h}$ full scale of data (Blaise et al., 2017). This explains why the PDFs of the pressure coefficients can be obtained so accurately over 6 orders of magnitude for the PDF (as seen thanks to the log-scale representation of the PDFs). This also allowed to decompose the measured signals into two main modes and one tail mode, this latter one being characterized by a very low weighting.

In practical applications, it is seldom possible to measure so long. It is therefore important to assess whether so long series are required for the de-mixing to be manageable. Fig. 9 shows the results of the de-mixing operation obtained for a time series of $10 \mathrm{~h}$ extracted from the complete set (a) and obtained with the whole data set (b). Identified mode parameters are represented in the Table on the right. These results show that a much shorter time series, with a duration that is similar to current practice in wind tunnels, provides virtually similar results. The main difference is the difficulty to identify the tail mode with the shorter time series. Indeed, the shorter duration does not allow to estimate the PDF of
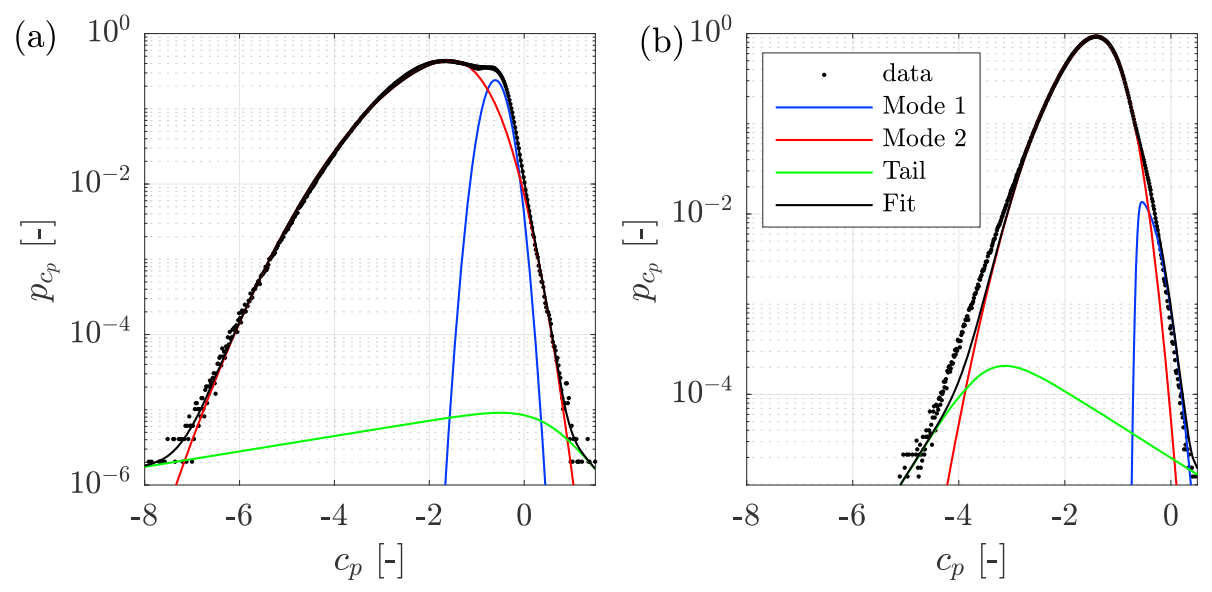

Fig. 7. PDF decomposition in mode 1, 2, tail (log scale): (a) tap 4 and (b) tap 8. 

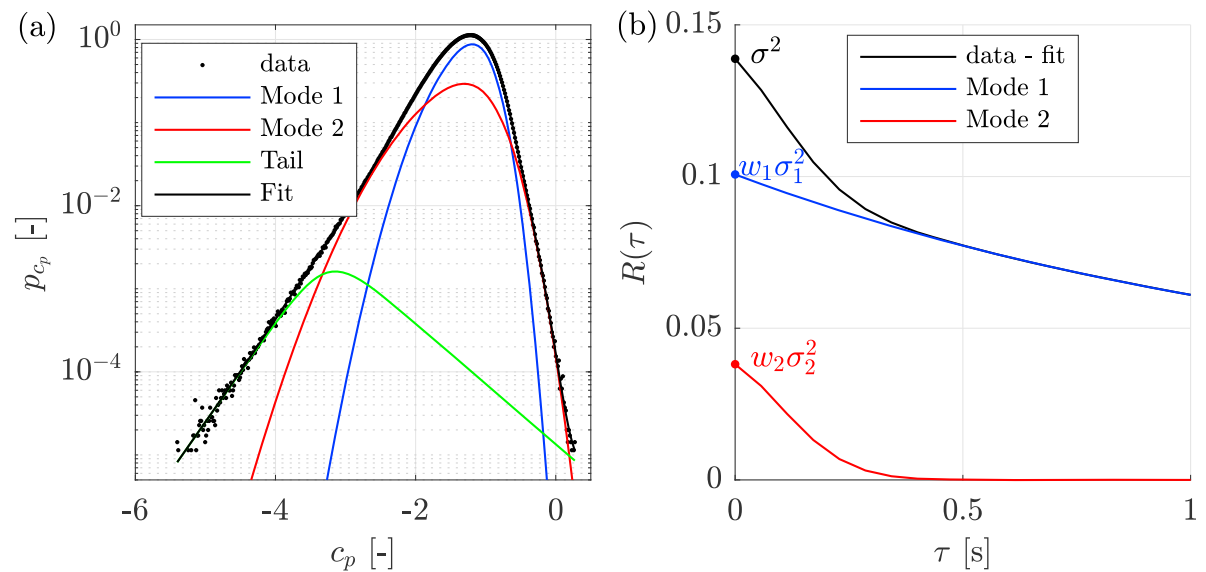

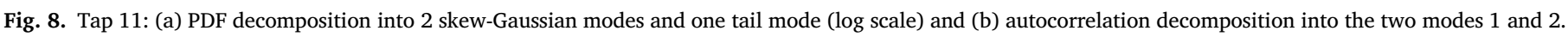
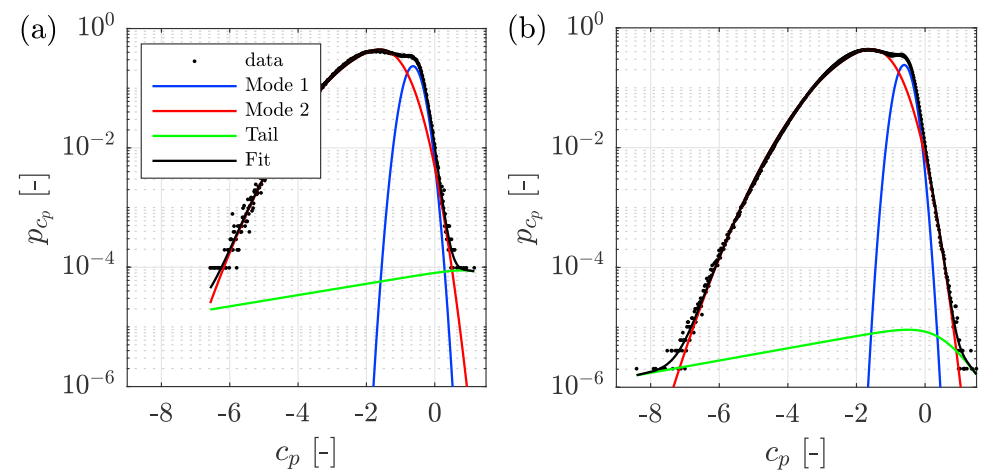

\begin{tabular}{c|c|c} 
(c) & $T=10 \mathrm{~h}$ & $T=370 \mathrm{~h}$ \\
\hline$w_{1}$ & 0.14 & 0.15 \\
$w_{2}$ & 0.86 & 0.85 \\
$\mu_{1}$ & -0.63 & -0.61 \\
$\mu_{2}$ & -1.93 & -1.89 \\
$\sigma_{1}$ & 0.23 & 0.21 \\
$\sigma_{2}$ & 0.82 & 0.82 \\
$\gamma_{3,1}$ & $4 \cdot 10^{-4}$ & $2 \cdot 10^{-8}$ \\
$\gamma_{3,2}$ & -0.63 & -0.61
\end{tabular}

Fig. 9. Tap 4: influence of duration measurement (full scale) in the de-mixing algorithm (a) $10 \mathrm{~h}$, (b) $370 \mathrm{~h}$ of measurement and (c) comparison of statistics of each mode.

the data below $10^{-4}$ while the complete data set provides a PDF estimate down to $10^{-6}$. A secondary difference concerns the skewness of the first mode $\gamma_{3,1}$ which is relatively different, but close to zero, which indicates a nearly Gaussian mode.

\section{Application 1: extreme values of pressure coefficients falling outside the monotone region}

\subsection{Cubic translation and peak factor estimation}

A usual way to estimate peak factors from pressure measurement uses cubic translation of a Gaussian process $u$ to a non-Gaussian (softening) process $x$ and the Hermite moment-based model (Winterstein, 1987). A softening process is characterized by a wider tail in the distribution compared to a Gaussian one (i.e. with $\gamma_{e}=\gamma_{4}-3>0$ ). Most of the wind pressure coefficients measured on buildings and roofs are softening processes. The mean peak factor of a cubic translation of a Gaussian process is computed by Kareem et al. (Kareem and Zhao, 1994),

$$
\begin{aligned}
\mu_{g_{\text {Her }}} & =\kappa\left\{\left(\beta+\frac{\gamma}{\beta}\right)+h_{3}\left[\beta^{2}+2 \gamma-1+\frac{1.98}{\beta^{2}}\right]+h_{4}\left[\beta^{3}+3 \beta(\gamma-1)\right.\right. \\
& \left.\left.+\frac{3}{\beta}\left(\frac{\pi^{2}}{6}-\gamma+\gamma^{2}\right)+\frac{5.44}{\beta^{3}}\right]\right\}
\end{aligned}
$$

where $h_{3}, h_{4}, \kappa$ are coefficients of moment-based Hermite model (Kwon and Kareem, 2011), $\gamma \simeq 0.5772$ is Euler' s constant and

$$
\begin{aligned}
& \beta=\sqrt{2 \ln \left(\nu_{0} T\right)}, \quad \nu_{0}=\sqrt{\frac{m_{2}}{m_{0}}}, \\
& m_{i}=\int_{-\infty}^{+\infty} n^{i} S_{x}(\omega) \mathrm{d} \omega, \quad h_{3}=\frac{\gamma_{3}}{4+2 \sqrt{1+1.5 \gamma_{4}}}
\end{aligned}
$$

$h_{4}=\frac{\sqrt{1+1.5 \gamma_{4}}-1}{18}, \quad \kappa=\frac{1}{\sqrt{1+2 h_{3}^{2}+6 h_{4}^{2}}}$

where $\nu_{0}$ is the mean zero up-crossing rate, $T$ is the duration, $m_{i}$ is the $i^{\text {th }}$ spectral moment of $x, S_{x}(\omega)$ is the power spectral density of $x$. The validity range of the Hermite model is limited to the so-called monotonic region, i.e. corresponding to a monotone transformation of $x(u)$, requiring $\mathrm{d} x / \mathrm{d} u>0$. Practically, this condition corresponds to (Kwon and Kareem, 2011; Winterstein and Mackenzie, 2011)

$\gamma_{e} \geq\left(1.25 \gamma_{3}\right)^{2}$

Pressure coefficients falling outside the monotone region are not strictly eligible to apply the cubic translation method. This is unfortunate since this method is simple and offers a straightforward estimation of the statistics of peak factors of non-Gaussian processes. Several solutions have been proposed to deal with the pressure coefficients falling outside the monotone region. Choi (Choi and Sweetman, 2010) proposes to overcome this limit by taking a softening-hardening-softening transformation in order to keep an increasing (monotone) transformation $x(u)$ and cross the decreasing part of the softening process. This technique 
requires making a hybrid model depending on the value of $x$ and deform the nature of the process. Peng (Peng et al., 2014) has studied another solution which consists in mapping vertically, horizontally or differently the points falling outside the domain, and apply peak factor formulae (12) with adjusted skewness and kurtosis.

The decomposition of the pressure coefficient into two components is useful to provide an alternative solution to this problem. This is indeed clear when noticing that the mixing of two (nearly) Gaussian distributions can eventually fall outside the monotone region. Since one is only interested in the statistics of a tail distribution (either left or right), the extreme values can be determined with the sole process of interest. Laid differently, it turns out that the de-mixing operation tends to provide two (or more) less non-Gaussian processes than the original signal. It is therefore suggested to de-mix the recorded pressure coefficient, keep the component of interest (largest succion peaks in this case) and, if the monotone condition is met, apply the cubic translation model on this component only. This suggestion is similar to what is proposed by Ding and Chen (2014).

\subsection{Extreme values estimation using the proposed de-mixing}

Fig. 10 shows a cross-plot $\left(\gamma_{3}, \gamma_{e}\right)$ of the 121 pressure coefficients measured on the roof. Light-gray symbols are used to indicate the pressure coefficients falling inside the monotone region, given by (14), while darker symbols correspond to pressure coefficients falling outside the monotone region. These pressure coefficients are also identified by black patches on the left. It is straightforward to link the location of major corner vortices to the pressure coefficients failing to meet the monotone condition.

It is also very interesting to notice that, after the de-mixing operation, the two decomposed modes have skewness and kurtosis falling inside the monotone region. These are represented in Fig. 10(b-c), where only the pressure coefficients which were not initially satisfying the monotone condition are shown. In fact, the monotone criterion is fulfilled for all taps after de-mixing: (i) Mode 1 represents the background turbulence and is almost Gaussian (low $\gamma_{3}$ and $\gamma_{e}$ ), see Fig. 10(b) while (ii) Mode 2 associated with corner vortices and larger succion peaks is more nonGaussian but still inside the monotone region, see Fig. 10(c).

Since the time series are long enough ( $370 \mathrm{~h}$ of full-scale data), it is possible to obtain accurate estimates of the peak factor by means of a conditional sampling technique, i.e. by dividing the time series into 10min windows, retaining the minimum peak pressure on each window, then average these values. This operation is performed for all 121 pressure coefficients in order to obtain a reference peak factor $\mu_{g}$.

The cubic translation method can be used with either the original method (including therefore the two modes), either with the second mode only. Pressure coefficients falling inside the monotone condition, represented with light-gray symbols, are not affected by the demixing. On this opposite, the extremes of pressure coefficients corresponding to the significantly non-Gaussian pressure, in the region affected by corner vortices, are poorly estimated with the cubic translation model applied on the full-time series (since it mixes two modes), see dark dots. However, when the cubic translation model is applied to the second mode, after de-mixing has been performed, we observe that the peak factors are much better estimated. This is illustrated in Fig. 10(d), where the points that are located the closest to the diagonal are the best estimated.

Although it goes beyond the scope of this paper, this concept could be extended to the determination of equivalent static wind loads with the help of the bi-cubic translation model (Blaise et al., 2016).

\section{Application 2: dynamic response of a cladding}

In structural design, façade elements are typically designed in a static manner and with the peak pressure acting on a given reference area. When the peak pressure is highly negative, application of this standard method might result in a very conservative design, since the peak pressure shall not be uniformly applied on the whole surface. A current trend to reduce the resulting loading to reasonable values is to evoke the limited extent in space of the peak event, shorter than the characteristic dimensions of the element. To do so requires very high spatial resolution and is difficult to realize in a wind tunnel in other circumstances than academic research (Amerio, 2017). Another track is followed here; indeed the above discussion about the mixing of several components, each one having its own timescale, might also provide another insight on this issue related to the design of façade elements. Instead of considering the spatial variation of the pressure field (which is anyways not homogenous in areas of interest), the short duration of the extreme events is invoked and we recommend to take advantage of the fact that a very large negative peak occurring during a very short period of time might less affect the structural response (depending on its modal features). Today, the design of large façade elements, extending sometimes to more than a dozen meters in span, results in natural frequencies reaching sometimes the lower bounds (typically $5 \mathrm{~Hz}$ ) prescribed in design codes. If the duration of the large suction peaks is much shorter than the natural period of the structural element, it can be seen as an impulsive loading. In this case, the response and therefore the severity of such an impulsive load scales with its momentum (dashed part of the time signal in Fig. 11) and not the magnitude of the force itself. This fact is well known from shock response spectra in structural dynamics (Clough and Penzien, 2003).

In order to further illustrate the concept, the shock response spectra of the pressure coefficients measured in the area affected by the corner vortices have been computed. They are simply obtained as the dynamic response of a mass-spring (undamped) system to the considered pressure coefficient applied in a homogenous way on a small-size façade element, using therefore the same pressure across the whole element. The response spectrum is sketched in Fig. 11(a) (right) by reporting the ratio between the maximum dynamic response over the maximum static response, as a function of the natural frequency $f$ of the cladding element (obtained by varying its stiffness). They are represented for the pressure coefficients measured at taps 6,9 and 11. They are represented as a function of the dimensionless frequency $f \tau_{2}$ where $\tau_{2} \equiv T_{x, 2}^{\star}$ corresponds to the timescale associated with the fast dynamics, i.e. a major result of the autocorrelation-based de-mixing method. When the PDF is bimodal, the shock response spectrum also presents an envelope with two modes: the slow turbulent background (large characteristic time, low frequency) and the fast shedding (short characteristic time, high frequency). Once the multiple timescale nature of the loading process is accepted, it is also possible to derive simple analytical solutions for the response. In particular, depending on the ratio of the natural period and the characteristic timescales identified in the loading process, it is possible that the slow turbulent process generates a background/resonant response, as is typical in buffeting analysis, while the fast shedding process generates an impulsive response that is governed by the momentum of the peaks in the signal. It is therefore recommended to separate the two components, treat the first one as a usual buffeting load and treat the second one by means of the statistics of the momentum corresponding to the peak pressures. One way to determine the statistics of the momentum is based on the segmentation of the signal based on a wavelet transform, in order to isolate peak events (Amerio, 2017) and determine the associated momentum. The full derivation of such approximate solutions goes beyond the scope of this paper. The most important point is that the extreme dynamic load (the fast loading component), provided it is identified as fast enough compared to the natural period of the cladding element, can be seen as an impulsive load. In this case, it is expected that a quasi-static design results in an over-safe design, as indicated in Fig. 11(b) for short characteristic durations $\tau_{2}$ of the fast loading component.

This approach could prove useful for small-size façade elements, which could not benefit from the imperfect correlation of pressure in space. For large elements, the coherence in space related to the fast mode 


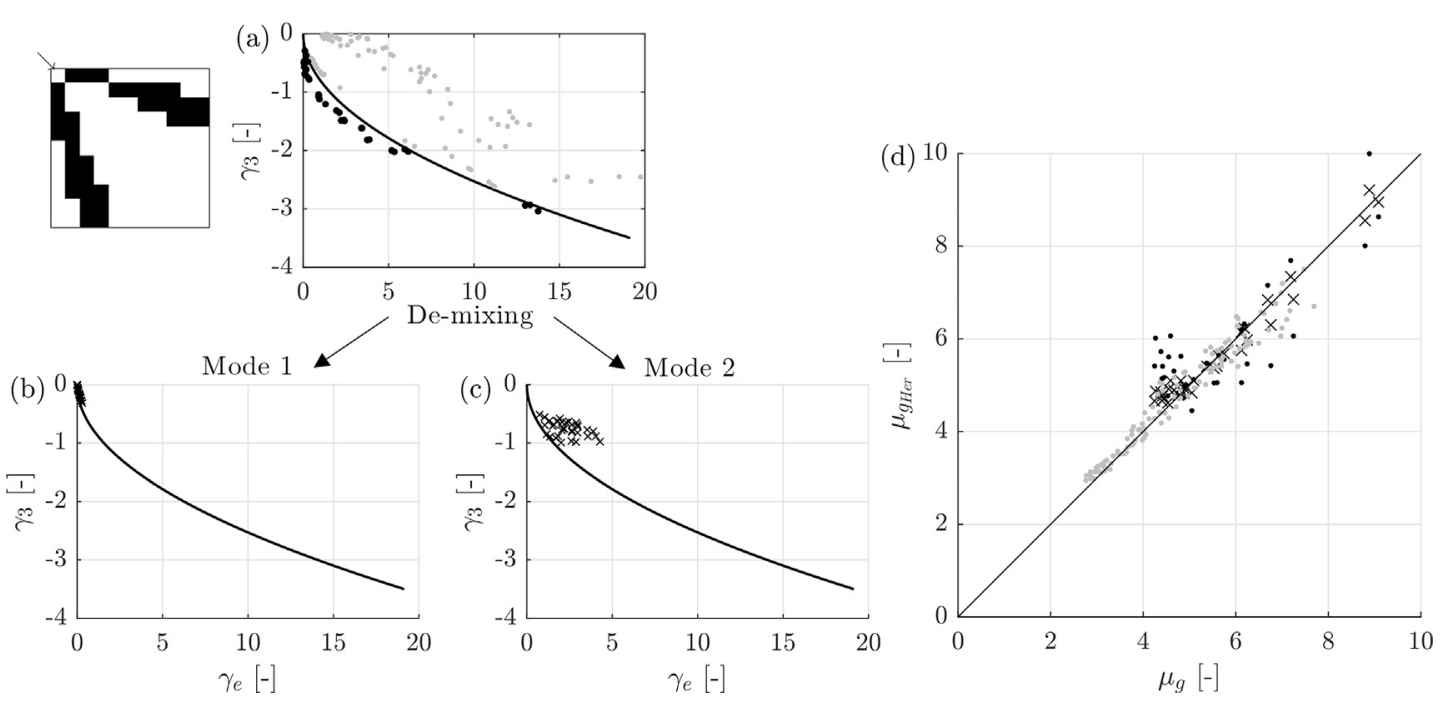

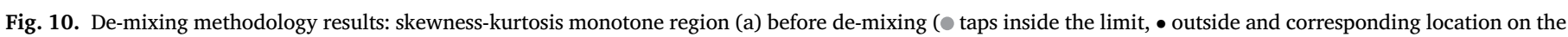

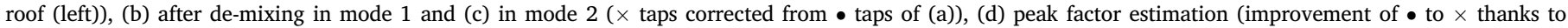
de-mixing).
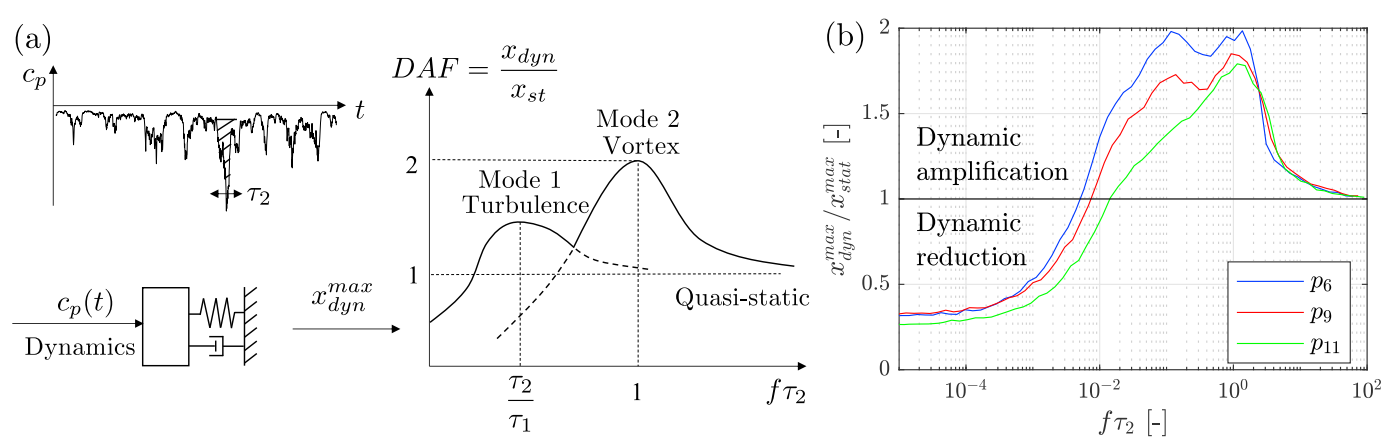

Fig. 11. (a) Dynamic response of a cladding and shock response function model, (b) application to taps 6 , 9 and 11 .

is crucial and the consideration of a reduced pressure, invoking the shorter timescale of the loading than the natural period, is not sufficient. In that case, a time-space analysis seems to be welcome. This question is unfortunately still not resolve today (Amerio, 2017) and is also deemed to go beyond the scope of this paper.

\section{Discussion and conclusions}

It is important to be able to distinguish the two (or more, if any) different components in the measured pressure since they correspond to different physics. To do so, a de-mixing method based on the autocorrelation of pressure coefficients has been proposed. Contrary to the similar methods based on a PDF only, this method is able to take advantage of the different timescales corresponding to the mixed components in the recorded pressure coefficients to provide a more robust demixing, especially in case where the classical de-mixing based on only a PDF struggles.

Because the method is applied to random processes, and not random variables (such a yearly extreme wind velocities), a specific derivation for the mixing has been developed, see Appendix. In particular, the dynamics of the mixing process $w(t)$ plays an important role.

The method has been successfully applied to the pressure coefficients measured on a flat root with a wind incidence that privileges corner vortices. The proposed algorithm has been used to identify two skewGaussian modes, and in some cases, an additional tail mode. Although the pressure coefficients have been analyzed separately, the results of the de-mixing show a very consistent distribution in space. This indicates that the mixing between the two physical phenomena smoothly evolves in space. This makes the flow field, in areas of interest, non-stationary in space, i.e. non-homogenous.

Finally, we have discussed two possible opportunities to apply the demixing algorithm. These applications are just briefly touched since they are based on the general idea to de-mix the measured signals into two (or more) components and are not specific to the use of the autocorrelation to assist the de-mixing. They concern, on the one hand, the use of the cubic translation model to estimate peak factors. By applying the existing peak factor for mildly non-Gaussian processes, it is seen that (i) the monotone condition is more easily met by the de-mixed components than the mixed-times series, (ii) the extreme values predicted by the model, when applied to a single component only, correlate much better with non-parametric statistical estimators. On the other hand, by bringing into the analysis the different timescales of the underlying phenomena, it is suggested that the design of façade elements is based on the superposition of a slow turbulent process and a fast impulsive mode corresponding to the fast and large succion peaks. Application to real cases require the determination of a shock response spectrum, as shown in Fig. 11.

\section{Declaration of competing Interest}

The authors declare that they have no known competing financial interests or personal relationships that could have appeared to influence 
the work reported in this paper.

\section{Acknowledgments}

The authors want to thank the Belgian National Fund for Scientific

\section{Appendix A: equations details}

The mixture process model

$x(t)=w_{1}(t) x_{1}(t)+w_{2}(t) x_{2}(t)$

is used to model the mixture between two pressure coefficients. Symbol $x$ is used to denote a pressure coefficient, while symbol $p$ is used to denote a PDF.

\subsection{The mixing processes}

The mixing processes $w_{1}(t)$ and $w_{2}(t)$ are discrete random processes. They take values in the set $\{0,1\}$ and are such that $w_{1}(t)+w_{2}(t)=1, \forall t>0$. At first rank, they are completely characterized by the probability functions $P_{W_{1}}(i, t)$ and $P_{W_{2}}(i, t)$ where the argument $i \in\{0,1\}$, so that, for instance, $P_{W_{1}}(1, t)$ represents the probability that $w_{1}(t)$ is equal to 1 at time $t$. We also assume that the mixing processes are stationary, i.e. the probability functions $P_{W_{1}}(i, t)$ and $P_{W_{2}}(i, t)$ do not explicitly depend on time. Because they take values in the set $\{0,1\}$, the average values of the mixing processes are simply given by

$\bar{w}_{i}=\sum_{i \in\{0,1\}} i P_{W_{i}}(i)=P_{W_{i}}(1)$

At second rank, they are completely characterized by the second rank probability functions $P_{W_{l}}\left(i, t ; i^{\prime}, t^{\prime}\right), P_{W_{2}}\left(i, t ; i^{\prime}, t^{\prime}\right)$ and $P_{W_{1} W_{2}}\left(i, t ; i^{\prime}, t^{\prime}\right)$ where the arguments $\left\{i, i^{\prime}\right\} \in\{0,1\}$. The processes are also assumed to be stationary in the wide sense (Papoulis, 1991), so that $P_{W_{1}}, P_{W_{2}}$ and $P_{W_{1} W_{2}}$ are explicitly functions of $\left|t-t^{\prime}\right|$ and not $t$ and $t^{\prime}$ separately. Accordingly, they can be written $P_{W_{1}}\left(i, i^{\prime}, t-t^{\prime}\right), P_{W_{2}}\left(i, i^{\prime}, t-t^{\prime}\right)$ and $P_{W_{1} W_{2}}\left(i, i^{\prime}, t-t^{\prime}\right)$. The meaning of $P_{W_{1}}\left(i, i^{\prime}, t-t^{\prime}\right)$ is the probability that $w_{1}\left(t_{0}\right)=i$. and $w_{1}\left(t_{0}+t-t^{\prime}\right)=i$ ' for any $t_{0}$. Same interpretations hold for $P_{W_{2}}$ and for the cross probability function $P_{W_{1} W_{2}}$. They are three functions of two arguments, but because $w_{1}(t)+w_{2}(t)=1$, meaning that $w_{2}=0$ when $w_{1}=1$ and vice versa, they are related to each other through

$$
\begin{aligned}
& P_{W_{1}}(1,1, \Delta t)=P_{W_{2}}(0,0, \Delta t)=P_{W_{1} W_{2}}(1,0, \Delta t)=\mathbb{E}\left[w_{1}\left(t_{0}\right) w_{1}\left(t_{0}+\Delta t\right)\right]=\bar{w}_{1}^{2}+R_{w}(\Delta t) \\
& P_{W_{1}}(1,0, \Delta t)=P_{W_{2}}(0,1, \Delta t)=P_{W_{1} W_{2}}(1,1, \Delta t)=\mathbb{E}\left[w_{1}\left(t_{0}\right)\left(1-w_{1}\left(t_{0}+\Delta t\right)\right)\right]=\bar{w}_{1} \bar{w}_{2}-R_{w}(\Delta t) \\
& P_{W_{1}}(0,1, \Delta t)=P_{W_{2}}(1,0, \Delta t)=P_{W_{1} W_{2}}(0,0, \Delta t)=\mathbb{E}\left[\left(1-w_{1}\left(t_{0}\right)\right) w_{1}\left(t_{0}+\Delta t\right)\right]=\bar{w}_{1} \bar{w}_{2}-R_{w}(\Delta t) \\
& P_{W_{1}}(0,0, \Delta t)=P_{W_{2}}(1,1, \Delta t)=P_{W_{1} W_{2}}(0,1, \Delta t)=\mathbb{E}\left[\left(1-w_{1}\left(t_{0}\right)\right)\left(1-w_{1}\left(t_{0}+\Delta t\right)\right)\right]=\bar{w}_{2}^{2}+R_{w}(\Delta t)
\end{aligned}
$$

where $R_{w}(\Delta t)=\sum_{i \in\{0,1\}} \sum_{i \in\{0,1\}}\left(i-\bar{w}_{1}\right)\left(i^{\prime}-\bar{w}_{1}\right) P_{W_{1}}\left(i, i^{\prime}, \Delta t\right)$ the autocorrelation function of $w_{1}(t)$. Notice that for a given realization of $w_{1}(t)$ is could be estimated by assuming that $w_{1}(t)$ is ergodic, i.e. $R_{w_{1}}(\Delta t)=\mathbb{E}\left[\left(w_{1}\left(t_{0}\right)-\bar{w}_{1}\right)\left(w_{1}\left(t_{0}+\Delta t\right)-\bar{w}_{1}\right)\right]$. This assumption is, however, not required for the following developments. The following results will be expressed as a function of the mean square correlation functions defined by

$Q_{1}(\Delta t)=\bar{w}_{1}^{2}+R_{w}(\Delta t) \quad ; \quad Q_{12}(\Delta t)=\bar{w}_{1} \bar{w}_{2}-R_{w}(\Delta t) \quad ; \quad Q_{2}(\Delta t)=\bar{w}_{2}^{2}+R_{w}(\Delta t)$

Because $R_{w}(0)=\sigma_{w_{1}}^{2}=\sigma_{w_{2}}^{2}=\bar{w}_{1} \bar{w}_{2}$ and $\lim _{\Delta t \rightarrow+\infty} R_{w}(\Delta t)=0$, the following limiting behaviors are observed

$$
\begin{gathered}
Q_{i}(0)=\bar{w}_{i}^{2}+\sigma_{w_{i}}^{2}=\bar{w}_{i} ; \quad Q_{12}(0)=\bar{w}_{1} \bar{w}_{2}-\sigma_{w_{1}}^{2}=0 \\
\lim _{\Delta t \rightarrow+\infty} Q_{i}(\Delta t)=\bar{w}_{i}^{2} ; \quad \lim _{\Delta t \rightarrow+\infty} Q_{12}(\Delta t)=\bar{w}_{1} \bar{w}_{2}
\end{gathered}
$$

Similar developments can be derived for higher ranks. They are not provided here since the proposed de-mixing method is limited to second order.

\subsection{The two underlying processes}

The two underlying processes $x_{1}(t)$ and $x_{2}(t)$ are continuous stationary random processes. At first rank, they are completely characterized by their PDF $p_{X_{1}}\left(x ; \pi_{1}\right)$ and $p_{X_{2}}\left(x ; \pi_{2}\right)$ where the explicit dependence on the model parameters $\pi_{1}$ and $\pi_{2}$ is clear. Notice they do not depend on time because of stationarity. The meaning of $p_{X_{1}}\left(x ; \pi_{1}\right) \mathrm{d} x$ is the probability that $x_{1}(t) \in[x, x+\mathrm{d} x]$ for any time $t$. Same interpretation holds for $p_{X_{2}}\left(x ; \pi_{2}\right) \mathrm{d} x$. The two processes $x_{1}(t)$ and $x_{2}(t)$ are also assumed to be independent, which translates into the factorization of their joint PDF. The average and standard deviation of these two random processes are noted $\mu_{1}, \mu_{2}$ and $\sigma_{1}, \sigma_{2}$. They are related to the PDF by means of the following definitions

$\mu_{i}=\int_{-\infty}^{+\infty} x p_{X_{i}}\left(x ; \pi_{i}\right) \mathrm{d} x \quad ; \quad \sigma_{i}^{2}=\int_{-\infty}^{+\infty}\left(x-\mu_{i}\right)^{2} p_{X_{i}}\left(x ; \boldsymbol{\pi}_{i}\right) \mathrm{d} x$.

At second rank, they are completely characterized by their second rank PDFs, $p_{X_{1}}\left(x, x^{\prime}, \Delta t ; \pi_{1}\right)$ and $p_{X_{2}}\left(x, x^{\prime}, \Delta t ; \pi_{2}\right)$. We notice that

$p_{X_{1} X_{2}}\left(x, x^{\prime}, \Delta t ; \boldsymbol{\pi}_{1}, \boldsymbol{\pi}_{2}\right)=p_{X_{1}}\left(x ; \boldsymbol{\pi}_{1}\right) p_{X_{2}}\left(x^{\prime} ; \boldsymbol{\pi}_{2}\right)$ 
since $p_{X_{1} X_{2}}\left(x, x^{\prime}, \Delta t ; \pi_{1}, \pi_{2}\right) \mathrm{d} x \mathrm{~d} x^{\prime}$ represents the probability that $x_{1}\left(t_{0}\right) \in[x, x+\mathrm{d} x]$ and $x_{2}\left(t_{0}+\Delta t\right) \in\left[x^{\prime}, x^{\prime}+\mathrm{d} x^{\prime}\right]$, which are two independent events. 8.3. The mixture process $x(t)$

Under the assumptions stated above, the first rank PDF of the mixture process reads

$p_{X}(x)=P_{W_{1}}(1) p_{X_{1}}\left(x ; \boldsymbol{\pi}_{1} \mid w_{1}=1\right)+P_{W_{2}}(1) p_{X_{2}}\left(x ; \boldsymbol{\pi}_{2} \mid w_{1}=1\right)$.

It does not explicitly depend on time, since $w_{i}(t)$ and $x_{i}(t), i \in\{1,2\}$ are both stationary. Because of the independence of the underlying and mixture processes, and since $P_{W_{i}}(1)=\bar{w}_{i}$, see (16),

$p_{X}(x)=\bar{w}_{1} p_{X_{1}}\left(x ; \boldsymbol{\pi}_{1}\right)+\bar{w}_{2} p_{X_{2}}\left(x ; \boldsymbol{\pi}_{2}\right)$

The simplicity of this equation is at the root of the argumentation in favor of mixture processes. Indeed, if $w_{1}(t)$ and $w_{2}(t)$ were not selected in the set $\{0,1\}$ but well in the interval $[0 ; 1]$, the PDF of $X(t)$ would have been expressed by means of a convolution. Although still manageable at first rank, this would quickly turn into cumbersome equations at higher ranks.

With a bit of calculus (and on account that $\bar{w}_{1}+\bar{w}_{2}=1$ ), it is straightforward to obtain

$\mu_{x}=\int_{-\infty}^{+\infty} x p_{X}(x) \mathrm{d} x=\bar{w}_{1} \mu_{1}+\bar{w}_{2} \mu_{2}$

$\sigma_{x}^{2}=\int_{-\infty}^{+\infty}\left(x-\mu_{x}\right)\left(x-\mu_{x}\right)^{2} p_{X}(x) \mathrm{d} x=\bar{w}_{1} \sigma_{1}^{2}+\bar{w}_{2} \sigma_{2}^{2}+\bar{w}_{1} \bar{w}_{2}\left(\mu_{1}-\mu_{2}\right)^{2}$.

In a similar fashion, the second rank PDF of the mixture process is given by

$p_{X}\left(x, x^{\prime}, \Delta t\right)=P_{W_{1}}(1,1, \Delta t) p_{X_{1}}\left(x, x^{\prime}, \Delta t ; \pi_{1}\right)+P_{W_{1}}(1,0, \Delta t) p_{X_{1} X_{2}}\left(x, x^{\prime}, \Delta t ; \boldsymbol{\pi}_{1}, \boldsymbol{\pi}_{2}\right)$

$+P_{W_{1}}(0,1, \Delta t) p_{X_{1} X_{2}}\left(x^{\prime}, x, \Delta t ; \boldsymbol{\pi}_{1}, \boldsymbol{\pi}_{2}\right)+P_{W_{1}}(0,0, \Delta t) p_{X_{2}}\left(x, x^{\prime}, \Delta t ; \boldsymbol{\pi}_{2}\right)$.

It depends on one time shift $\Delta t$ only because the mixing and underlying processes are stationary in the wide sense. The autocorrelation function of $x(t)$, defined by

$R_{x}(\Delta t)=\iint_{-\infty}^{+\infty}\left(x-\mu_{x}\right)\left(x^{\prime}-\mu_{x}\right) p_{X}\left(x, x^{\prime}, \Delta t\right) \mathrm{d} x \mathrm{~d} x^{\prime}=\iint_{-\infty}^{+\infty} x x^{\prime} p_{X}\left(x, x^{\prime}, \Delta t\right) \mathrm{d} x \mathrm{~d} x^{\prime}-\mu_{x}^{2}$,

can be developed by substituting (25) and by considering the relations (17). After some simplifications, we obtain

$R_{x}(\Delta t)=Q_{1}(\Delta t)\left(\mu_{1}^{2}+R_{x_{1}}(\Delta t)\right)+Q_{2}(\Delta t)\left(\mu_{2}^{2}+R_{x_{2}}(\Delta t)\right)+2 Q_{12}(\Delta t) \mu_{1} \mu_{2}-\mu_{x}^{2}$

where the autocorrelation functions of the independent components are given by

$R_{x_{i}}(\Delta t)=\iint_{-\infty}^{+\infty}\left(x-\mu_{i}\right)\left(x^{\prime}-\mu_{i}\right) p_{X_{i}}\left(x, x^{\prime}, \Delta t ; \boldsymbol{\pi}_{i}\right) \mathrm{d} x \mathrm{~d} x^{\prime}$

for $i \in\{1,2\}$. Finally equation (27) can be rearranged as

$R_{x}(\Delta t)=Q_{1}(\Delta t) R_{x_{1}}(\Delta t)+Q_{2}(\Delta t) R_{x_{2}}(\Delta t)+\left(\mu_{1}-\mu_{2}\right)^{2} R_{w}(\Delta t)$.

It is possible to check that the value at the origin is given by

$R_{x}(0)=\bar{w}_{1} \sigma_{1}^{2}+\bar{w}_{2} \sigma_{2}^{2}+\bar{w}_{1} \bar{w}_{2}\left(\mu_{1}-\mu_{2}\right)^{2}$

on account of the limiting behaviors given in (18), which is well consistent with the expression (24) given for the variance of $x(t)$.

Equation (29) shows that the timescales in $x(t)$ result from an intricate mix of the timescales in the mixing processes $w_{1}(t)$ and $w_{2}(t)$, through $Q_{1}$ and $Q_{2}$, as well as the timescales in $x_{1}(t)$ and $x_{2}(t)$. There are two particular cases of this general expression, which are of practical relevance.

When the characteristic correlation time $T_{w}^{\star}$ in $w_{1}(t)$ and $w_{2}(t)$ is much shorter than the correlation time $T_{x}^{\star}$ in $x_{1}(t)$ and $x_{2}(t)$, the mixing processes are fast (in the limiting case there correspond to delta-correlated noise and the random process boils down to the known mixture model for random variables, where the specific arrangement in time in the process does not matter anymore). In that case, for $T_{w}^{\star} \ll \Delta t$, we notice that $Q_{i}(\Delta t) \rightarrow \bar{w}_{i}^{2}$, see (18) and for $\Delta t \ll T_{x}^{\star}$, we notice that $R_{x_{i}}(\Delta t) \rightarrow \sigma_{i}^{2}$, so that the autocorrelation function of $x(t)$ admits to following two asymptotic cases

$R_{x}^{T_{w}^{\star} \ll T_{x}^{\star}}(\Delta t) \sim\left\{\begin{array}{cc}\bar{w}_{1}^{2} R_{x_{1}}(\Delta t)+\bar{w}_{2}^{2} R_{x_{2}}(\Delta t) & \text { for } T_{w}^{\star} \ll T_{x}^{\star} \sim \Delta t \\ Q_{1}(\Delta t) \sigma_{1}^{2}+Q_{2}(\Delta t) \sigma_{2}^{2}+\left(\mu_{1}-\mu_{2}\right)^{2} R_{w}(\Delta t) & \text { for } \Delta t \sim T_{w}^{\star} \ll T_{x}^{\star}\end{array}\right.$.

We notice that the value at the origin in $R_{x}^{T_{w}^{\star} \ll T_{x}^{\star}}(\Delta t)$, extrapolated from the case $T_{w}^{\star} \ll \Delta t^{\sim} T_{x}^{\star}$ (which is in principle not authorized), is equal to $\bar{w}_{1}^{2} \sigma_{1}^{2}+$ $\bar{w}_{2}^{2} \sigma_{2}^{2}$ and is different from $\sigma_{x}^{2}$. This is a noticeable difference between the mixture process and the mixture (random variable) model. 
On the opposite, when the characteristic correlation time $T_{w}^{\star}$ in $w_{1}(t)$ and $w_{2}(t)$ is much longer than the correlation time $T_{x}^{\star}$ in $x_{1}(t)$ and $x_{2}(t)$, the mixing process is a slow process meaning that many samples are taken in one random variable before switching to the other. In this case, for $\Delta t \ll T_{w}^{\star}$, we notice that $Q_{i}(\Delta t) \rightarrow \bar{w}_{i}$ and $R_{w}(\Delta t) \rightarrow \bar{w}_{1} \bar{w}_{2}$, see (18) and the autocorrelation function of $x(t)$ reads

$R_{x}^{T_{x}^{\star} \ll T_{w}^{\star}}(\Delta t) \sim \bar{w}_{1} R_{x_{1}}(\Delta t)+\bar{w}_{2} R_{x_{2}}(\Delta t)+\bar{w}_{1} \bar{w}_{2}\left(\mu_{1}-\mu_{2}\right)^{2}$

for $\Delta t^{\sim} T_{x}^{\star} \ll T_{w}^{\star}$. The other limiting case, related to $T_{x}^{\star} \ll T_{w}^{\star \sim} \Delta t$, is not exploitable since $R_{x_{1}}, R_{x_{2}}$ and $R_{w}$ asymptotically tend to zero as $T_{x}^{\star} \ll \Delta t$.

\section{References}

Amerio, L., 2017. Experimental High Resolution Analysis of the Pressure Peaks on a Building Scale Model Facades. Politecnico di Milano. PhD thesis.

Amerio, L., Allsop, A., Pomaranzi, G., Zasso, A., 2018. Experimental assessment of the current techniques for the assessment of the area- averaged wind-pressure using wind tunnel pressure data. In: Proceedings of the UK Wind Engineering Society Conference. Leeds, UK.

Azzalini, A., Valle, A.D., 1996. The multivariate skew-normal distribution. Biometrika 83 (4), 715-726.

Baker, C.J., 2000. Aspects of the use of proper orthogonal decomposition of surface pressure fields. Wind Struct. 3 (2), 97-115.

Banks, D., Meroney, R.N., Sarkar, P.P., Zhao, Z., Wu, F., 2000. Flow visualization of conical vortices on flat roofs with simultaneous surface pressure measurement. J. Wind Eng. Ind. Aerod. 84, 65-85.

Bergh, H., Tijdeman, H., 1967. Theoretical and Experimental Results for the Dynamic Response of Pressure Measuring Systems.

Best, R.J., Holmes, J.D., 1983. Use of eigenvalues in the covariance integration method for determination of wind load effects. J. Wind Eng. Ind. Aerod. 13 (1-3), 359-370.

Bienkiewicz, B., Tamura, Y., Ham, H.J., Ueda, H., Hibi, K., 1995. Proper orthogonal decomposition and reconstruction of multichannel roof pressure. J. Wind Eng. Ind. Aerod. 54, 369-381.

Blaise, N., Denoël, V., 2013. Principal static wind loads. J. Wind Eng. Ind. Aerod. 113, 29-39.

Blaise, N., Canor, T., Denoël, V., 2014. Calculation of third order joint acceptance function for line-like structures. In: Proceedings of the XIII Conference of the Italian Association for Wind Engineering.

Blaise, N., Canor, T., Denoël, V., 2016. Reconstruction of the envelope of non-Gaussian structural responses with principal static wind loads. J. Wind Eng. Ind. Aerod. 149, 59-76.

Blaise, N., Andrianne, T., Denoël, V., 2017. Assessment of extreme value overestimations with equivalent static wind loads. J. Wind Eng. Ind. Aerod. 168, 123-133.

Carassale, L., 2012. Scale effects on the buffeting response of wind-excited structures. In: Meccanica dei Materiali e delle Strutture, pp. 41-48. Palermo, Italy.

Carassale, L., Brunenghi, M.M., 2012. Identification of meaningful coherent structures in the wind-induced pressure on a prismatic body. J. Wind Eng. Ind. Aerod. 104, 216-226.

Chen, X., Huang, G., 2009. Evaluation of peak resultant response for wind-excited tall buildings. Eng. Struct. 31, 858-868, 04.

Choi, M., Sweetman, B., 2010. The hermite moment model for highly skewed response with application to tension leg platforms. Journal of Offshore Mechanics and Arctic Engineering-transactions of The Asme 132, 05.

Clough, R.W., Penzien, J., 2003. Dynamics of Structures. Computers and Structures, Inc, berkeley. CA.

Comon, P., Jutten, C., 2010. Handbook of Blind Source Separation. Academic Press, Oxford.

Cook, N.J., 1986. Designers Guide to Wind Loading of Building Structures. Part 1, 1.

Cook, N.J., 2016. Short communication: on the Gaussian-exponential mixture model for pressure coefficients. J. Wind Eng. Ind. Aerod. 153, 71-77, 06.

Davenport, A.G., 1964a. Note on the distribution of the largest value of a random function with application to gust loading. Proc. Inst. Civ. Eng. 28 (2), 187-196.

Davenport, A.G., June 1964. The buffeting of large superficial structures by atmospheric turbulence. Ann. N. Y. Acad. Sci. 116, 135-160.

Denoël, V., 2011. On the background and biresonant components of the random response of single degree-of-freedom systems under non-Gaussian random loading. Eng. Struct. 33 (8), 2271-2283.

Denoël, V., Maquoi, R., 2012. The concept of numerical admittance. Arch. Appl. Mech. 82 (10-11), 1337-1354.

Ding, J., Chen, X., 2014. Assessment of methods for extreme value analysis of nonGaussian wind effects with short-term time history samples. Eng. Struct. 80, 75-88.

Dyrbye, C., Hansen, S.O., 1996. Wind Loads on Structures. john wiley \& sons, Chichester/ New York/Brisbane/Toronto/Singapore.

Eurocode, 1991. en 1991-1-4: Actions on structures-general actions-wind actions. european committee for standardization.

Gharib, M., Rambod, E., Shariff, K., 1998. A universal time scale for vortex ring formation. J. Fluid Mech. 360, 121-140.

Gioffre, M., Grigoriu, M., 2005. Simulation of strongly non-Gaussian wind pressures using mixed models. In: Safety and Reliability of Engineering Systems and Structures ICOSSAR 2005. Rome, Italy.

Gioffre, M., Grigoriu, M., 2016. Mixed models for highly skewed wind pressures. In: XIV Conference of the Italian Association for Wind Engineering, IN-VENTO-2016. Terni, Italy.
Gioffrè, M., Gusella, V., Grigoriu, M., 2001. Non-Gaussian wind pressure on prismatic buildings. i: stochastic field. J. Struct. Eng. 127 (9), 981-989.

Grigoriu, Mircea, 1998. Simulation of stationary non-Gaussian translation processes. J. Eng. Mech. 124 (2), 121-126.

Gurley, K., Kareem, A., 1999. Simulation of Non-gaussian Processes. Computational Stochastic Mechanics, CSM98. Spanos.

Holmes, J.D., 1981. Non-Gaussian characteristics of wind pressure fluctuations. J. Wind Eng. Ind. Aerod. 7 (1), 103-108.

Holmes, J.D., 1997. Equivalent time averaging in wind engineering. J. Wind Eng. Ind. Aerod. 72, 411-419 (Selected papers from the Ninth International Symposium on Wind Engineering).

Holmes, J.D., Sankaran, R., Kwok, K.C.S., Syme, M.J., 1997. Eigenvector modes of fluctuating pressures on low-rise building models. J. Wind Eng. Ind. Aerod. 71, 697-707. Yu923.

Huang, M., Huang, S., Feng, H., Lou, W., 2016. Non-Gaussian time-dependent statistics of wind pressure processes on a roof structure. Wind and Structures An International Journal 23 (10), 275-300.

Huang, Guoqing, Luo, Ying, Yang, Qingshan, Tian, Yuji, 2017. A semi-analytical formula for estimating peak wind load effects based on hermite polynomial model. Eng. Struct. 152, 856-864.

Hunt, J.C.R., Vassilicos, J.C., Cambridge University Press, Isaac Newton Institute for Mathematical Sciences, Vassilicos, D.A.M.T.P.J.C., 2000. Turbulence Structure and Vortex Dynamics. Cambridge University Press.

Jolliffe, Ian, 2005. Principal component analysis. Encyclopedia of Statistics in Behavioral Science. John Wiley \& Sons, Ltd.

Kareem, A., Zhao, J., 1994. Analysis of non-Gaussian surge response of tension leg platforms under wind loads. J. Offshore Mech. Arctic Eng. 116, 08.

Kareem, A., Zhou, Y., 2003. Gust loading factor?past, present and future. J. Wind Eng. Ind. Aerod. 91 (12-15), 1301-1328.

Kawai, H., Nishimura, G., 1996. Characteristics of fluctuating suction and conical vortices on a flat roof in oblique flow. J. Wind Eng. Ind. Aerod. 60, 211-225. The Wind Engineering Society's 2nd UK Conference.

Kho, S., Baker, C., Hoxey, R., 2002. Pod/arma reconstruction of the surface pressure field around a low rise structure. J. Wind Eng. Ind. Aerod. 90 (12-15), 1831-1842.

Kollu, S., Rayapudi, R., Narasimham, S., Pakkurthi, K.M., 2012. Mixture probability distribution functions to model wind speed distributions. International Journal of Energy and Environmental Engineering 3 (1), 27.

Kwon, D.K., Kareem, A., 2009. Peak factor for non-Gaussian processes revisited. In: The Seventh Asia-Pacific Conference on Wind Engineering. Taipei, Taiwan, November 8-12.

Kwon, D.K., Kareem, A., 2011. Peak factors for non-Gaussian load effects revisited. J. Struct. Eng. 137 (12), 1611-1619.

Lawson, T.V., 1980. Wind effects on buildings. In: Lawson, T.V. (Ed.), Design Applications, vol. 1. Applied Science Publishers, Ltd, London.

Letchford, C.W., Mehta, K.C., 1993. The distribution and correlation of fluctuating pressures on the Texas-tech building. J. Wind Eng. Ind. Aerod. 50 (1-3), 225-234.

Lin, J.-X., Surry, D., Tieleman, H.W., 1995. The distribution of pressure near roof corners of flat roof low buildings. J. Wind Eng. Ind. Aerod. 56, 235-265.

Liu, M., Chen, X., Yang, Q., 2017. Estimation of peak factor of non-Gaussian wind pressures by improved moment-based hermite model. J. Eng. Mech. 143 (7), 06017006.

Newberry, C.W., Eaton, K.J., Mayne, J.R., Building Research Establishment, 1974. Wind loading on tall buildings : further results from Royex house. Watford : building research establishment. Published in Industrial Aerodynamics Abstracts 4 (No. 4). July-August 1973.

Papoulis, A., 1991. Probability, Random Variables, and Stochastic Processes, thrid edit edition. McGraw Hill, New York.

Peng, X., Yang, L., Gavanski, E., Gurley, K., Prevatt, D., 2014. A comparison of methods to estimate peak wind loads on buildings. J. Wind Eng. Ind. Aerod. 126, 11-23.

Peterka, J.A., 1983. Selection of local peak pressure coefficients for wind tunnel studies of buildings. J. Wind Eng. Ind. Aerod. 13 (1), 477-488.

Quan, Y., Gu, M., Tamura, Y., Chen, B., 2009. An extreme-value estimating method of non-Gaussian wind pressure. In: Proceedings of the 7th Asia-Pacific Conference on Wind Engineering. Taipei, Taiwan, November 8-12.

Rigo, F., 2017. Unsteady Pressure Measurement Around Aerodynamic Bodies: Development of a Calibration Apparatus and Wind Tunnel Testing. Master's thesis. University of Liège.

Rigo, F., Andrianne, T., Denoël, V., 2018. Mixture model in high-order statistics for peak factor estimation on low-rise building. In: Proceedings of the 15th Conference of the Italian Association for Wind Engineering. Naples, Italy.

Rizzo, F., Barbato, M., Sepe, V., 2018. Peak factor statistics of wind effects for hyperbolic paraboloid roofs. Eng. Struct. 173, 313-330.

Sadek, F., Simiu, E., 2002. Peak non-Gaussian wind effects for database-assisted low-rise building design. J. Eng. Mech. 128 (5), 530-539. 
Shields, M.D., Deodatis, G., Bocchini, P., 2011. A simple and efficient methodology to approximate a general non-Gaussian stationary stochastic process by a translation process. Probabilist. Eng. Mech. 26 (4), 511-519.

Solari, G., Carassale, L., Tubino, F., 2007. Proper orthogonal decomposition in wind engineering. part 1: a state-of-the-art and some prospects. Wind Struct. 10 (2), 153-176.

Tubino, F., Solari, G., 2005. Double proper orthogonal decomposition for representing and simulating turbulence fields. Journal of Engineering Mechanics-Asce 131 (12), 1302-1312.
Winterstein, S., 1987. Moment-based Hermite Models of Random Vibration. Lyngby.

Winterstein, S., Mackenzie, C., 2011. Extremes of nonlinear vibration: comparing models based on moments. 1-moments, and maximum entropy 135.

Yu, J., Chen, K., Mori, J., Rashid, M.M., 2013. A Gaussian mixture copula model based localized Gaussian process regression approach for long-term wind speed prediction. Energy 61, 673-686. 